\title{
Editorial
}

\section{INDEXACIÓN DE REVISTAS CIENTÍFICAS.}

En el año 1963 la Organización para la Cooperación y Desarrollo Económicos (OCDE) creó el manual de Frascatti ${ }^{1}$; que es un documento técnico que contiene las definiciones básicas y categorías de las actividades de investigación y desarrollo (I+D) ; con la finalidad de medir y comparar entre países los recursos asignados a $(\mathrm{I}+\mathrm{D})$. Este documento también plantea una taxonomía de las áreas científicas y tecnológicas, dentro de las cuales el grupo tres corresponde a Ciencias Médicas, contiene a la Medicina básica, la Medicina clínica (ej. Odontología) y Ciencias de la Salud.

En la década de 1970; el gran aumento de publicaciones científicas tanto en ciencias médicas como en ciencias de la salud, ha generado la necesidad de decantar las publicaciones que realmente se consideran de buena calidad, respecto de otras. En el caso de la investigación en ciencias de la Salud se ha auto regulado mediante la utilización de una estrategia de búsqueda de la mejor información científica, denominado Medicina Basada en Evidencias ${ }^{2}$. Sin embargo con la globalización de internet muchas iniciativas de publicación han surgido en la red en todo el mundo, algunas no han tenido sostenibilidad en el tiempo y otras no cumplen con las recomendaciones del Comité Internacional de Editores de Revistas Médicas (ICJME) ${ }^{3}$, organismo que tiene el objetivo de brindar las recomendaciones para supervisar la práctica y los estándares éticos en la publicaciones biomédicas. Una publicación del 2003 da cuenta que solo el $15 \%$ de publicaciones son útiles para el cuidado y atención de pacientes, a lo cual le deberíamos llamar el impacto real. ${ }^{4}$.

En ese contexto surge la necesidad de valorar las publicaciones, de acuerdo a procedimientos estandarizados, con la finalidad de que los usuarios sepan a que atenerse al momento de ubicar y seleccionar un artículo para respaldar su práctica clínica. La indexación de revistas tiene esta finalidad, la de ser un filtro de las publicaciones, tanto en periodicidad, vigencia, calidad y visibilidad.

\footnotetext{
${ }^{1}$ OCDE. Propuesta de Norma Práctica para Encuestas de Investigación y Desarrollo Experimental. 6ta Edición.2002.

${ }^{2}$ Forrest JL, Miller SA. Evidence-Based decision making in action: Part 1 -Finding the Best Clinnical Evidence. J contemp Dent Pract. 2002 Feb15;4(1):42-52

${ }^{3}$ Atención primaria. Requisitos de uniformidad para manuscritos presentados para publicación en revistas biomédicas. Vol 21. Num 5. Marzo; 1998

${ }^{4}$ Forrest JL, Miller SA. Evidence-Based decision making in action: Part 2 -Evaluating and applying the clinnical evidence. J contemp Dent Pract. 2003 Feb15;4(1):42-52
} 
No se debe confundir la indexación con el factor de impacto, en tanto este último es un cálculo matemático de la importancia de una publicación científica en el medio académico, no hace referencia al uso clínico de esa información. El Factor de Impacto fue creado por el instituto para la información científica (ISI) ${ }^{5}$. Entre los portales de internet que indexan revistas científicas tenemos LATINDEX, LILACS, DOAJ, SCIELO, Scopus, PubMed, el año 2017; la revista Odontología Activa se prepara para postular a la Indexación, esperando lograr ese objetivo el año 2018, presentamos el volumen 2 número 3.

Dr. Ebingen Villavicencio Caparó

Director de la Revista

\footnotetext{
${ }^{5}$ Alger J. El Factor de Impacto en las Revistas Científicas. Primera reunión de editores de revistas científicas nacionales. Disponible en http://www.bvs.hn/Honduras/pdf/FactorImpacto.pdf
} 